\title{
MdWRKY11 improves copper tolerance by directly promoting the expression of the copper transporter gene MdHMA5
}

\author{
Kun Shi', Xuan Liu', Yunpeng Zhu', Yixue Bai', Dongqian Shan ${ }^{1}$, Xiaodong Zheng' ${ }^{1}$, Lin Wang ${ }^{1}$, Haixia Zhang ${ }^{1}$, \\ Chanyu Wang', Tianci Yan', Fangfang Zhou',2, Zehui Hu', Yanzhao Sun', Yan Guo ${ }^{2}$ and Jin Kong ${ }^{1}$
}

\begin{abstract}
Overuse of fungicides and fertilizers has resulted in copper (Cu) contamination of soils and toxic levels of Cu in apple fruits. To breed Cu-resistant apple (Malus domestica) cultivars, the underlying molecular mechanisms and key genes involved in $\mathrm{Cu}$ resistance must be identified. Here, we show that MdWRKY11 increases Cu tolerance by directly promoting the transcription of MdHMA5. MdHMA5 is a Cu transporter that may function in the storage of excess $\mathrm{Cu}$ in root cell walls and stems for Cu tolerance in apple. The transcription factor MdWRKY11 is highly induced by excess $\mathrm{Cu}$. MdWRKY11 overexpression in transgenic apple enhanced $\mathrm{Cu}$ tolerance and decreased $\mathrm{Cu}$ accumulation. Apple calli transformed with an MdWRKY11-RNAi construct exhibited the opposite phenotype. Both an in vivo chromatin immunoprecipitation assay and an in vitro electrophoretic mobility shift assay indicated that MdWRKY11 binds to the promoter of MdHMA5. Furthermore, MdWRKY11 promoted MdHMA5 expression in transgenic apple plants, as revealed by quantitative PCR. Moreover, inhibition of MdWRKY11 expression by RNA interference led to a significant decrease in MdHMA5 transcription. Thus, MdWRKY11 directly regulates MdHMA5 transcription. Our work resulted in the identification of a novel MdWRKY11-MdHMA5 pathway that mediates Cu resistance in apple.
\end{abstract}

\section{Introduction}

Apple (Malus domestica) is one of the four most widely cultivated fruit crop species, and ensuring that apples do not accumulate toxic levels of metals from the soil is important for public health ${ }^{1,2}$. The widespread use of the Bordeaux fungicide mixture, farmyard manure containing $\mathrm{Cu}$ as fertilizer, and wastewater for irrigation has led to the accumulation of excess $\mathrm{Cu}$ in the soil and in apple fruits ${ }^{1}$. Indeed, the $\mathrm{Cu}$ levels of apples in many orchards have been reported to be ten times higher than safe limits $^{3,4}$, and the problem is getting worse. The threat to human health from toxic, $\mathrm{Cu}$-contaminated apple fruits is a long-term problem because it removing excess $\mathrm{Cu}$ already present in soils is challenging ${ }^{5}$.

Correspondence: Jin Kong (jinkong@cau.edu.cn)

${ }^{1}$ College of Horticulture, China Agricultural University, 100193 Beijing, China ${ }^{2}$ College of Biological Sciences, China Agricultural University, 100193 Beijing, China
An extreme excess amount of $\mathrm{Cu}$ in the soil leads to leaf chlorosis, limits apple tree growth, and greatly reduces yield $^{6}$; however, light-to-moderate $\mathrm{Cu}$ pollution of orchard soils, which does not cause these symptoms, probably poses a greater threat to human health, as toxic, $\mathrm{Cu}$ contaminated apple fruits can continue to be produced by trees that show no signs of $\mathrm{Cu}$ stress, causing the problem to go undetected ${ }^{1,7}$. Therefore, it is important to elucidate the molecular mechanisms underlying the response to excess $\mathrm{Cu}$ in apple both for monitoring $\mathrm{Cu}$ contamination and for molecular breeding of $\mathrm{Cu}$-resistant apple cultivars.

Excess $\mathrm{Cu}$ inhibits photosystem II activity and photosynthesis, impairs the elongation of roots and shoots, reduces fruit quality and yield, and can trigger senescence and death ${ }^{8-11}$. To withstand excess $\mathrm{Cu}$ in the soil, plants have developed two strategies for maintaining normal $\mathrm{Cu}$ levels in their tissues: $\mathrm{Cu}$ efflux and $\mathrm{Cu}$ sequestration ${ }^{12}$. When excess $\mathrm{Cu}$ enters root epidermal cells, the first 
strategy is to export $\mathrm{Cu}$ back outside the plant cytoplasm, possibly via storage in the root cell wall ${ }^{12,13}$. The second strategy is to store excess $\mathrm{Cu}$ in tissues that are less sensitive to the toxic effects of $\mathrm{Cu}$, such as stem tissue ${ }^{12}$. In this second case, in chelated form, $\mathrm{Cu}$ moves up the stem through the transpiration stream after being transported into xylem ${ }^{14}$. Chelation not only decreases the cytosolic free $\mathrm{Cu}$ concentration, thereby reducing photosystem II damage, but also facilitates $\mathrm{Cu}$ transport through the plant $^{15}$. Once free $\mathrm{Cu}$ is chelated by metallothionein or phytochelatin proteins, metallochaperones deliver the $\mathrm{Cu}$-ligand complexes directly to $\mathrm{P}_{1 \mathrm{~B}}$-type ATPases for transport $^{16,17}$.

Heavy Metal ATPase 5 (HMA5) is a $\mathrm{Cu}$-specific $\mathrm{P}_{1 \mathrm{~B}^{-}}$ type ATPase that transports chelated $\mathrm{Cu}$ across membranes ${ }^{18}$. HMA 5 is involved in $\mathrm{Cu}$ tolerance in two ways: it transports $\mathrm{Cu}$ out of the root, and it mediates $\mathrm{Cu}$ uploading for long-distance transport and redistribution within the plant. In the $\mathrm{Cu}$ export strategy, HMA5 in the plasma membrane of root epidermal cells transports excess $\mathrm{Cu}$ out of the cytoplasm to maintain proper $\mathrm{Cu}$ levels in the plant ${ }^{19}$. AtHMA5, which has been identified as component of a QTL in Arabidopsis thaliana, transports chelated $\mathrm{Cu}$ outside root epidermal cells under $\mathrm{Cu}$ excess stress ${ }^{20,21}$. The Arabidopsis hma5 mutant is hypersensitive to excess $\mathrm{Cu}$ and accumulates relatively large amounts of $\mathrm{Cu}$ in its roots ${ }^{16}$. A similar function was reported for SvHMA5II in Silene vulgaris ${ }^{21}$. With respect to the redistribution strategy, chelated $\mathrm{Cu}$ moves laterally from cell to cell via HMA5 transporters and is ultimately uploaded to the xylem for transport from the roots to the stem $^{22,23}$. The $\mathrm{Cu}$ insensitivity of the stem makes this tissue an ideal place to sequester excess $\mathrm{Cu}$ away from $\mathrm{Cu}$-sensitive organs such as roots and leaves ${ }^{19,23}$. In rice (Oryza sativa), OsHMA5 is localized in the plasma membrane of root pericycle cells, where it loads $\mathrm{Cu}$ into the xylem for long-distance transport to stems ${ }^{22,24}$. However, there have not been any reports of HMA5 genes in woody plant species, in which the $\mathrm{Cu}$ resistance mechanism is expected to be even more complex.

Transcription factors (TFs) play a central role in the response to excess heavy metal by orchestrating several physiological processes ${ }^{25-28}$. There have been several reports on the transcriptional regulation of the $\mathrm{Cu}$ response in multicellular eukaryotes. The transcription factors SPL7, CRR1, and Ace1-like protein regulate the $\mathrm{Cu}$ chaperones $\mathrm{CCH}$ (involved in $\mathrm{Cu}$ chelation and detoxification), COPT1 (involved in $\mathrm{Cu}$ absorption), and $\mathrm{FeSOD}$ and $\mathrm{Cu} / \mathrm{ZnSOD}$ (involved in reactive oxygen species mitigation), respectively $^{29-31}$. However, the transcriptional regulation of HMA5 under excess $\mathrm{Cu}$ remains unknown.

WRKY TFs play a critical role in the response to excess heavy metals (iron, cadmium, and aluminum) by regulating their chelation and translocation of the metals and by reducing secondary oxidative damage ${ }^{32-34}$. WRKYs belong to one of the largest TF families in plants and are named for their highly conserved WRKYGQK heptapeptide at the Nterminus, which specifically binds to W-box cis-elements (containing a TTGACC/T core sequence) in the promoters of downstream target genes ${ }^{35-37}$. However, it is not known whether WRKY TFs are involved in the response to excess $\mathrm{Cu}$ or what regulatory pathways might be involved.

In this study, we isolated $M d W R K Y 11$, which is significantly induced in response to $\mathrm{Cu}$ stress, in apple. Overexpression of MdWRKY11 conferred increased Cu tolerance to transgenic apple trees. Furthermore, we demonstrated that MdWRKY11 directly binds to the promoter of MdHMA5, which encodes a $\mathrm{P}_{1 \mathrm{~B}}$ ATPase, and activates its expression. MdHMA5 functions in $\mathrm{Cu}$ transport and decreases $\mathrm{Cu}$ accumulation in apple plants. In addition to isolating a novel transcriptional regulatory pathway of $\mathrm{Cu}$ tolerance in plants, this study provides marker genes for monitoring $\mathrm{Cu}$ contamination in orchards.

\section{Results}

Expression of MdWRKY11 in response to $\mathrm{CuSO}_{4}$ treatment

To identify WRKY genes that might be involved in the response to excess $\mathrm{Cu}$, we screened the expression of 29 candidate MdWRKYS in the leaves and roots of hydroponic plants treated with $500 \mu \mathrm{M} \mathrm{CuSO}_{4}$. Among these $M d W R K Y \mathrm{~s}, M d W R K Y 11$ expression was significantly induced in response to $\mathrm{CuSO}_{4}$ treatment in both the roots and the leaves (Fig. 1a), suggesting that this gene has an important role in the response to excess $\mathrm{Cu}$. Therefore, we selected MdWRKY11 for further study.

\section{Subcellular localization of MdWRKY11}

To examine the subcellular localization of MdWRKY11, 35S::MdWRKY11-GFP was infiltrated into N. benthamiana leaves via Agrobacterium-mediated transient transformation. The MdWRKY11-GFP fluorescence was localized exclusively to the nucleus (Fig. 1b).

\section{Transcriptional activity of MdWRKY11}

The transcriptional activation activity of MdWRKY11 was assayed in a yeast system. Yeast cells transformed with $p B D-M d W R K Y 11$ or the positive control construct pCL-1 grew well on SD-Trp-His selective media and displayed $\alpha$-galactosidase activity, whereas yeast cells carrying the negative control construct pGBKT7 were unable to grow on the selective medium (Fig. 1c). These results indicate that MdWRKY11 is a transcriptional activator in the yeast system.

\section{$\mathrm{Cu}$ tolerance of transgenic apple plants overexpressing MdWRKY11}

To investigate the potential function of MdWRKY11 in $\mathrm{Cu}$ tolerance, transgenic apple plants overexpressing 

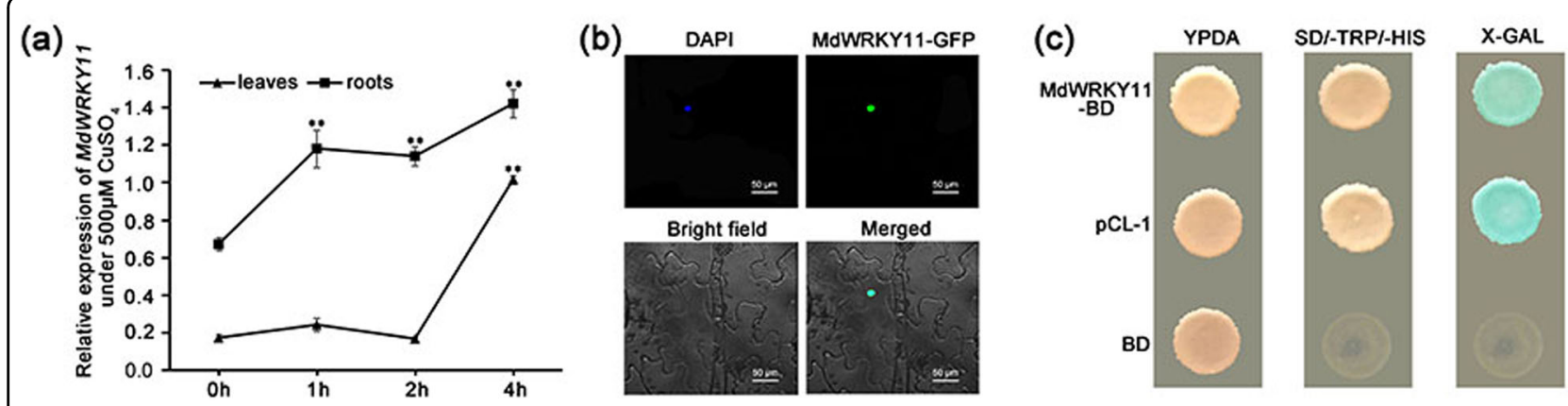

Fig. 1 Expression, subcellular localization, and transcriptional activity of MdWRKY11. a MdWRKY11 expression in the leaves and roots under excess Cu stress, as detected by qPCR. The MdWRKY11 expression level was normalized to the internal MdActin expression level. The apple plants were treated with $500 \mu \mathrm{M} \mathrm{CuSO}_{4}$ for $0,1,2$, and $4 \mathrm{~h}$. The data are the means \pm SDs of triplicate experiments for each time point. The asterisks indicate values that are significantly different from those of the control (Student's $t$-test): ${ }^{*} P<0.05$; ${ }^{* *} P<0.01$. $\mathbf{b}$ MdWRKY11-GFP is localized to the nucleus of Nicotiana benthamiana cells. 355::MdWRKY11-GFP was transiently expressed in epidermal cells of $N$. benthamiana leaves and visualized by confocal microscopy (×40). The nucleus was dyed with 4,6-diamidino-2-phenylindole (DAPI). c Transcriptional activation of MdWRKY11 in yeast cells. Yeast AH109 strains expressing $p C L-1$, binding domain $(B D)$, and pBD-MdWRKY11 were cultured on yeast peptone dextrose adenine agar (YPDA) or selective SD-His-Trp media. PCL-1 encoding the GAL4 protein and the empty vector PGBKT7 (BD) were used as the positive and negative controls, respectively

MdWRKY11 were generated via Agrobacterium-mediated transformation. The expression of MdWRKY11 in OEWRKY11-1, OEWRKY11-2, and OEWRKY11-3 transgenic apple lines was significantly higher than that in the untransformed controls (Fig. S1a). Therefore, we selected these three lines for further analysis.

The control apple plants grew slowly under excess $\mathrm{Cu}$ conditions. After thirty days of $\mathrm{Cu}$ treatment, the older leaves displayed chlorosis and brown spots, and the newer leaves turned yellow. However, these toxic symptoms were not observed in the transgenic plants (Fig. 2a). Therefore, the overexpression of MdWRKY11 conferred enhanced $\mathrm{Cu}$ tolerance to the transgenic apple plants.

We also examined MdWRKY11 expression and $\mathrm{Cu}$ tolerance in transgenic apple calli harboring either the overexpression construct or an MdWRKY11 RNA interference construct. MdWRKY11 overexpression or underexpression was confirmed by qPCR (Fig. S1b). Similar to that which occurred for the plants transformed with the overexpression construct, transgenic apple calli overexpressing MdWRKY11 presented enhanced $\mathrm{Cu}$ tolerance. Calli in which MdWRKY11 expression had been decreased by the RNAi construct presented decreased $\mathrm{Cu}$ tolerance (Fig. 2b). Under normal conditions, the control calli and both types of transgenic calli appeared to grow at similar rates. In the presence of $\mathrm{CuSO}_{4}$, however, calli overexpressing MdWRKY11 grew better than the control, whereas calli carrying the RNAi construct grew more slowly. Overall, MdWRKY11 overexpression resulted in increased $\mathrm{Cu}$ tolerance, while decreased MdWRKY11 expression resulted in decreased $\mathrm{Cu}$ tolerance.
Effects of MdWRKY11 overexpression on $\mathrm{Cu}$ accumulation in the roots and leaves of transgenic apple plants

To further investigate the role of MdWRKY11 in $\mathrm{Cu}$ tolerance, we used X-ray fluorescence (XRF) microtomography to analyze the content and distribution of $\mathrm{Cu}$ in control plants and MdWRKY11-overexpressing plants treated with excess $\mathrm{Cu}$. The same pattern of $\mathrm{Cu}$ distribution was observed in both the control and transgenic apple plants. The highest $\mathrm{Cu}$ level was in the vascular cylinder (VC). The $\mathrm{Cu}$ level decreased with increasing distance from the $\mathrm{VC}$, being highest in the endodermis (EN) and lowest in the epidermis (EP). Consistent with their $\mathrm{Cu}$-tolerant phenotype, the $M d W R K Y 11$-overexpressing plants had significantly less $\mathrm{Cu}$ than did the control plants in their roots and leaves (Fig. 3).

\section{Binding of MdWRKY11 to the MdHMA5 promoter and its effect on MdHMA5 transcription}

To determine how MdWRKY11 overexpression provides increased $\mathrm{Cu}$ tolerance, we analyzed the expression of key genes involved in $\mathrm{Cu}$ absorption and transport (Fig. $4 \mathrm{a}$ and S2). Among these genes, the expression of $M d H M A 5$, which encodes a $\mathrm{Cu}$-specific transporter, was significantly higher in the MdWRKY11-overexpressing lines than in untransformed control plants (Fig. 4a). Similarly, MdHMA5 expression in transgenic calli overexpressing MdWRKY11 was nearly 50\% higher than that in control calli. Conversely, MdHMA5 expression decreased to nearly half the control levels in calli transformed with the MdWRKY11 RNAi construct (Fig. S4a). 

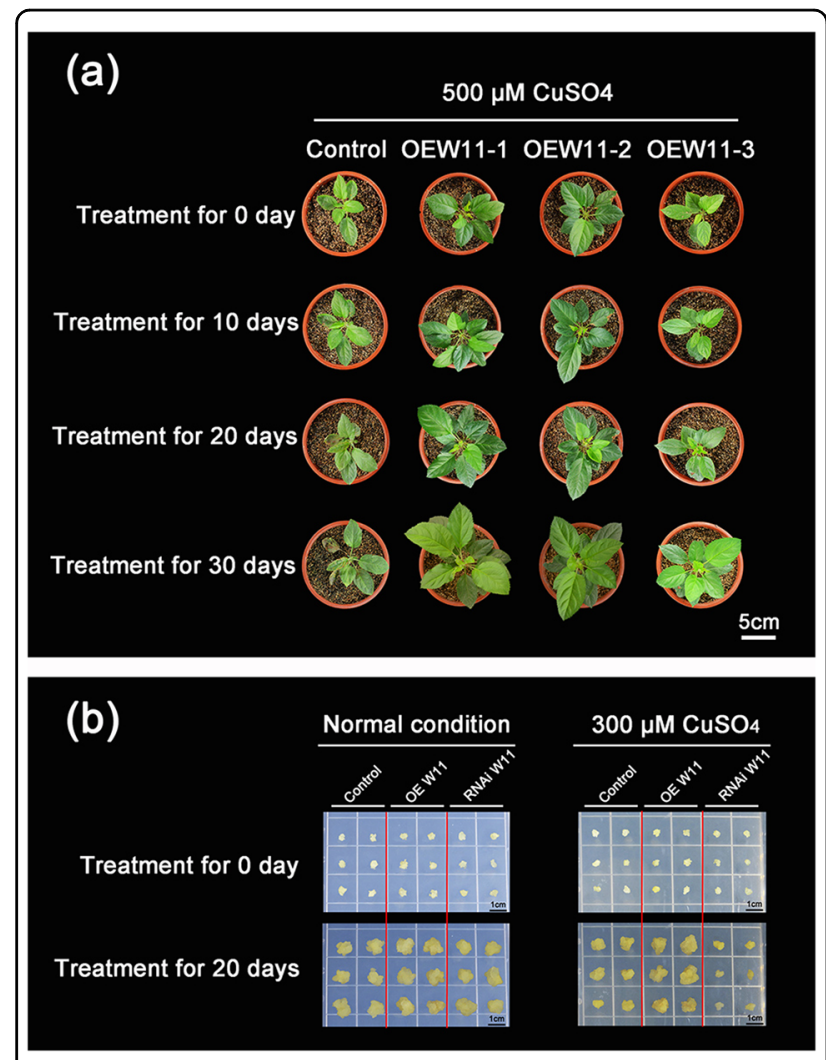

Fig. 2 Assessment of $\mathrm{Cu}$ tolerance of transgenic apple plants and calli subjected to $\mathrm{CuSO}_{4}$ treatment. a Phenotypes of three

transgenic apple lines overexpressing MdWRKY11 and an untransformed control plant treated with $500 \mu \mathrm{M} \mathrm{CuSO}_{4}$ for 10, 20, and 30 days. $\mathbf{b}$ Cu tolerance of transgenic MdWRKY11-overexpressing and MdWRKY11 RNAi calli and control calli cultured on media supplemented with excess $\mathrm{Cu}\left(300 \mu \mathrm{M} \mathrm{CuSO}_{4}\right)$ or normal $\mathrm{Cu}$ concentrations for 20 days

These results suggest that MdWRKY11 positively regulates the expression of MdHMA5.

To test whether MdHMA5 is directly regulated by MdWRKY11, we examined whether MdWRKY11 binds to the MdHMA5 promoter both in vivo and in vitro using chromatin immunoprecipitation (ChIP)-qPCR and EMSAs, respectively. ChIP-qPCR analysis showed that the P2 fragment, which contains the W-box motif of the MdHMA5 promoter, was enriched in samples from the transgenic lines (Fig. 4b), confirming that MdWRKY11 binds specifically to the MdHMA5 promoter in vivo. EMSAs demonstrated the binding of MdWRKY11 to the P2 fragment of the MdHMA5 promoter in vitro. This binding was reduced in a dose-dependent manner with the addition of a 100-fold or 200-fold excess of unlabeled competitor. In addition, the binding was completely abolished when the probe contained a mutated W-box element, further confirming that the W-box of the P2 fragment of the MdHMA5 promoter is the binding site for MdWRKY11 (Fig. 4c).

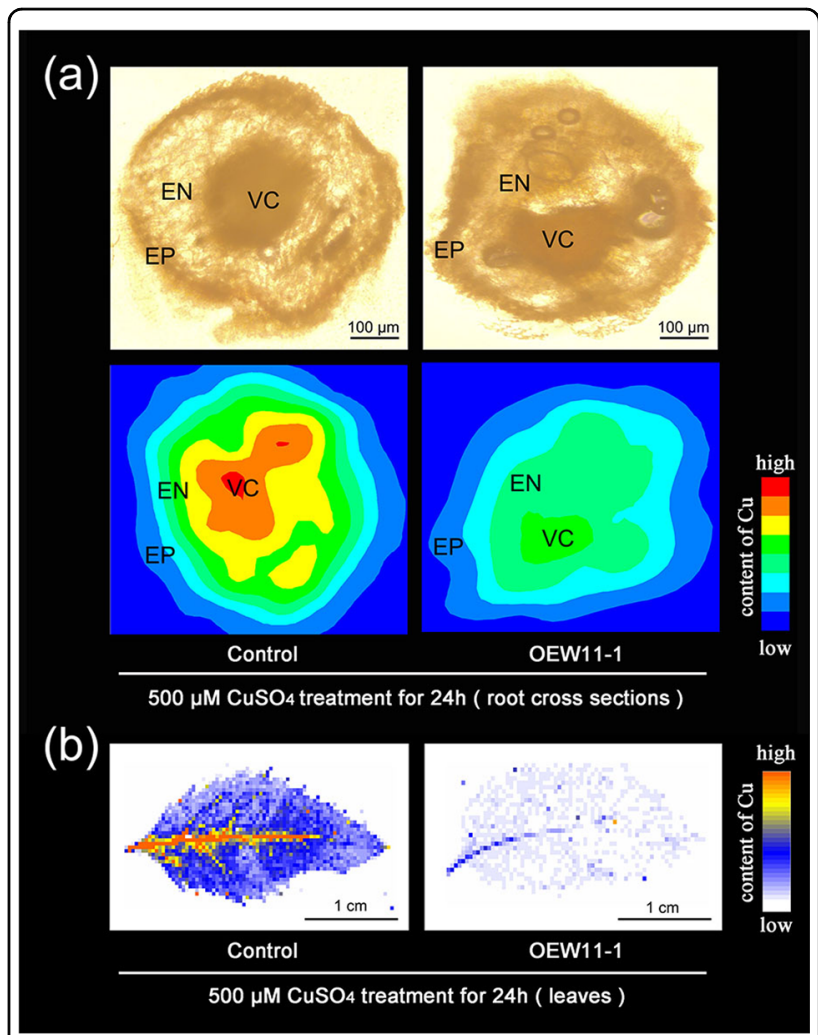

Fig. $3 \mathrm{Cu}$ levels and distribution shown as $\mu$-XRF elemental maps of the roots and leaves of transgenic plants overexpressing MdWRKY11 and an untransformed control plant treated with $\mathbf{5 0 0 \mu \mathrm { M } \mathrm { CuSO }}{ }_{4}$ for $\mathbf{2 4} \mathbf{~ h}$. a Cu level and distribution depicted as $\mu-$ XRF elemental maps of the roots. VC vascular cylinder, EN endodermis, EP epidermis. b Cu level and distribution shown as $\mu$-XRF elemental maps of leaves

We further tested the effects of MdWRKY11 on MdHMA5 expression in apple calli transiently cotransformed with an MdWRKY11 overexpression construct and with a construct in which GUS expression was driven by the MdHMA5 promoter. The ratio of GUS to LUC activity was significantly higher in calli expressing proMdHMA5::GUS, 35S::MdWRKY11-GFP, and 35S::LUC than in control calli without the 35S::MdWRKY11-GFP construct (Fig. 4d). Taken together, these results indicate that MdWRKY11 specifically binds to the MdHMA5 promoter and activates its expression.

\section{Phylogenetic analysis, subcellular localization, and functional analysis of MdHMA5}

Phylogenetic analysis showed that MdHMA5 is more closely related to AtHMA5 (Fig. 5a), which plays an important role in $\mathrm{Cu}$ homeostasis and detoxification in Arabidopsis ${ }^{16,21}$, than to other HMA5s from other species. MdHMA5 expression was significantly induced by excess $\mathrm{CuSO}_{4}$ treatment in both the roots and leaves of hydroponic plants (Fig. 5b). Similar to AtHMA5, 
MdHMA5 was localized to the plasma membrane ${ }^{16,21}$ (Fig. 5c). These results suggest that, like AtHMA5, MdHMA5 may be involved in the detoxification of excess $\mathrm{Cu}$.

To investigate the function of MdHMA5 in $\mathrm{Cu}$ detoxification, apple calli were transformed with $M d H M A 5$ overexpression or MdHMA5-RNAi constructs and cultured on media containing normal or excess amounts of $\mathrm{Cu}$ (Fig. 5d). All of the calli grew well under normal conditions, with no significant differences. On media supplemented with excess $\mathrm{Cu}$, however, the MdHMA5overexpressing calli grew markedly better than did the controls, while the growth of the MdHMA5-RNAi calli was more severely inhibited. Furthermore, XRF microtomography analysis showed that the highest $\mathrm{Cu}$ level occurred in MdHMA5-RNAi calli and that the lowest $\mathrm{Cu}$ level occurred in the MdHMA5-overexpressing calli (Fig. $5 e)$. These results indicate that the overexpression of MdHMA5 confers increased $\mathrm{Cu}$ tolerance to apple calli by maintaining the $\mathrm{Cu}$ level, while MdHMA5-RNAi calli exhibited the opposite phenotype. These results are in good agreement with the results of our analysis of transgenic apple plants overexpressing MdWRKY11.

\section{Discussion}

$\mathrm{Cu}$ contamination has become a severe problem in apple orchards by impairing growth and reducing apple yield. Importantly, human health may be threatened by toxic apple fruit production ${ }^{1}$. Understanding the mechanisms underlying $\mathrm{Cu}$ resistance in apple is the basis for molecular breeding of $\mathrm{Cu}$-resistant apple cultivars.

We screened apple WRKY genes to isolate the key transcription factor involved in $\mathrm{Cu}$ resistance. MdWRKY11 expression was significantly induced by excess $\mathrm{Cu}$ (Fig. 1a) and was chosen for subsequent research. MdWRKY11 is a typical Group II WRKY transcription factor; it is located in the nucleus and functions as a transcriptional activator in a yeast system (Fig. 1b, c). We overexpressed MdWRKY11 in Gala, a popular apple cultivar grown worldwide ${ }^{38}$, which conferred increased $\mathrm{Cu}$ tolerance to the transgenic plants. Moreover, a similar $\mathrm{Cu}$-tolerant phenotype was observed for transgenic apple calli overexpressing MdWRKY11, while calli transformed with an MdWRKY11 RNAi construct were less tolerant to excess $\mathrm{Cu}$ (Fig. 2b). These results imply that MdWRKY11 plays an important role in $\mathrm{Cu}$ resistance and constitute the first line of evidence that WRKY TFs are involved in regulating the response of apple to excess $\mathrm{Cu}$.

To isolate the genes regulated by MdWRKY11 as part of the $\mathrm{Cu}$ response, we measured the expression of key genes involved in $\mathrm{Cu}$ absorption and transport in control plants and MdWRKY11-overexpressing apple plants. MdHMA5 expression significantly increased in the plants overexpressing MdWRKY11 (Fig. 4a). The direct regulation of
MdHMA5 was indicated by in vivo ChIP-qPCR (Fig. 4b), by in vitro EMSAs (Fig. 4c) and by additional in vivo transgenic tests (Fig. 4d). In addition, MdHMA5 expression was relatively low in calli transformed with an MdWRKY11 RNAi construct (Fig. S2a), suggesting that MdWRKY11 plays a critical role in regulating the expression of MdHMA5. Taken together, these results demonstrate that MdWRKY11 binds to the MdHMA5 promoter to activate its transcription under excess $\mathrm{Cu}$ stress.

Given that the role of HMA5 in woody plant species is not known, we investigated its involvement in $\mathrm{Cu}$ detoxification in apple calli transformed with MdHMA5overexpression or MdHMA5-RNAi constructs. Under excess $\mathrm{Cu}$ conditions, overexpression of MdHMA5 provided enhanced $\mathrm{Cu}$ tolerance, while RNAi of MdHMA5 expression decreased $\mathrm{Cu}$ tolerance (Fig. $5 \mathrm{~d}$ ). This confirmed the important role of MdHMA5 in $\mathrm{Cu}$ detoxification.

To clarify how the MdWRKY11-MdHMA5 pathway functions in $\mathrm{Cu}$ resistance, we used XRF to analyze the concentration and distribution of $\mathrm{Cu}$ in transgenic apple plants and calli presenting altered MdWRKY11 or MdHMA5 expression (Fig. 5e). Under excess $\mathrm{Cu}$ conditions, transgenic apple plants overexpressing MdWRKY11 had markedly lower concentrations of $\mathrm{Cu}$ in both their roots and leaves compared with those of the controls (Fig. 3). The $\mathrm{Cu}$ level of the roots was much higher than that of the leaves in both the control plants and transgenic plants (Fig. S3b, d). Compared with those of the control plants, the roots of the transgenic plants had less $\mathrm{Cu}$ in every tissue layer, indicating that roots of the transgenic plants accumulate less $\mathrm{Cu}$ than do those of the control plants, which suggests that epidermal root cells might transport $\mathrm{Cu}$ outside the cytoplasm more efficiently, possibly through upregulated MdHAM5. In root cross-sections, the $\mathrm{Cu}$ level was highest in the $\mathrm{VC}$ and decreased from the endodermis to the epidermis in both the transgenic plants and control plants. These results imply that the MdWRKY11-induced MdHMA5 pathway may also be involved in $\mathrm{Cu}$ loading, as has been reported in rice ${ }^{24}$. Indeed, physiological studies showed that, compared with $\mathrm{Cu}$-sensitive apple rootstocks, $\mathrm{Cu}$-tolerant apple rootstocks had lower levels of $\mathrm{Cu}$ in their roots, possibly the result of more efficient $\mathrm{Cu}$ export or redistribution ${ }^{2}$.

Our observations of transgenic plants suggest that apple employs a redistribution strategy to detoxify excess $\mathrm{Cu}$. Furthermore, $\mathrm{Cu}$ levels were dramatically lower in transgenic calli overexpressing MdHMA5 and higher in MdHMA5-RNAi calli, indicating that MdHMA5 is also involved in $\mathrm{Cu}$ export. Therefore, MdHMA5, which is directly regulated by MdWRKY11 and functions in transmembrane $\mathrm{Cu}$ transport, is possibly required for the extrusion or redistribution of $\mathrm{Cu}$ in apple. This would 


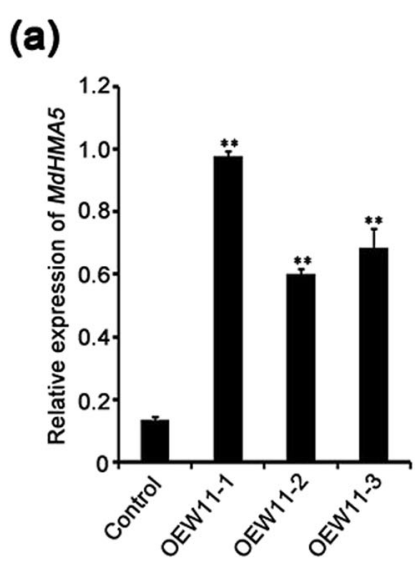

(c)

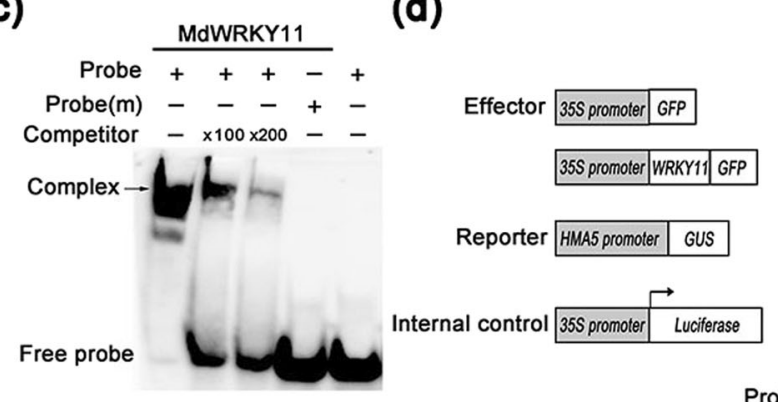

(b)

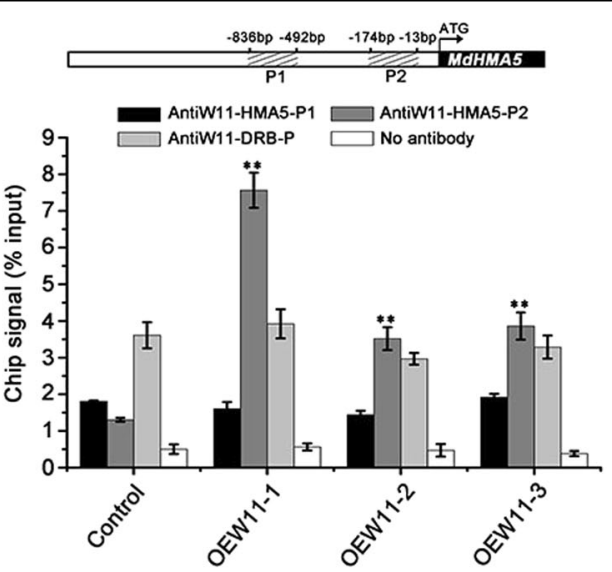

(d)

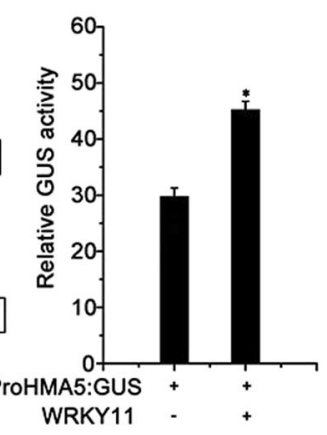

Fig. 4 MdWRKY11 binds to the promoter and activates the expression of MdHMA5, which encodes a Cu-transporting $\mathrm{P}_{1 \mathrm{~B}}$-type ATPase. a MdHMA5 expression in transgenic and control apple plants. b Binding of MdWRKY11 to the W-box of the MdHMA5 promoter indicated by chromatin immunoprecipitation (ChIP)-qPCR. The position of the W-box is indicated by the gray bar. The ChIP signal was quantified as the percentage of immunoprecipitated DNA out of the total input DNA, as determined by qPCR. A parallel experiment without antibodies and the promoter of MdDRB served as the negative control. c Electrophoretic mobility shift assay (EMSA) results confirming the in vitro binding of MdWRKY11 to the MdHMA5 promoter fragment. The arrow indicates the position of a protein-DNA complex after incubation with GST-MdWRKY11 and the biotin-labeled DNA probe MdHMA5. Both the probe containing a W-box and the probe $(\mathrm{m})$ containing a mutated W-box were synthesized according to the sequence of the MdHMA5 promoter. $\mathbf{d}$ Relative GUS activity normalized with respect to luciferase (LUC) activity in transiently transformed apple calli expressing 35S::MdWRKY11-GFP, proMdHMA5::GUS, and 35S::LUC; the relative GUS activity in transiently transformed apple calli expressing 355::GFP, proMdHMA5::GUS, and 35S::LUC served as the control. The data are the means \pm SDs of triplicate experiments. The asterisks indicate values that are significantly different from those of the control (Student's $t$-test): ${ }^{*} P<0.05 ;{ }^{* *} P<0.01$

explain the significantly lower $\mathrm{Cu}$ levels in the roots and leaves of the transgenic apple plants overexpressing MdWRKY11 compared with those of the controls. Together, our findings suggest that the use of the MdWRKY11-MdHMA5 pathway is a key strategy for $\mathrm{Cu}$ detoxification in apple.

Based on these observations, we present a model for $\mathrm{Cu}$ detoxification in apple (Fig. 6): excess $\mathrm{Cu}$ induces MdWRKY11, which directly binds to the promoter of MdHMA5 (a Cu-transporting $\mathrm{P}_{1 \mathrm{~B}}$-type ATPase gene), increasing its transcription. MdHMA5 then reduces $\mathrm{Cu}$ levels in the cytoplasm by increasing $\mathrm{Cu}$ transmembrane transport in root cells. Our work identified a novel MdWRKY11-MdHMA5 pathway that mediates $\mathrm{Cu}$ resistance in apple. This study not only contributes to the molecular breeding of Cu-resistant apple cultivars but also provides marker genes to monitor $\mathrm{Cu}$ contamination.

\section{Materials and methods}

\section{Plant materials and growth conditions}

Gala 3 (Malus $x$ domestica Borkh. cv. Royal Gala) plants were cultured on MS media containing $0.2 \mathrm{mg} \mathrm{l}^{-1}$ indole3 -acetic acid (IAA), $0.3 \mathrm{mg} \mathrm{l}^{-1}$ 6-benzylaminopurine (6BA), and $0.1 \mathrm{mg} \mathrm{l}^{-1}$ gibberellin 3 (GA3) at $23^{\circ} \mathrm{C}$ under a $16 \mathrm{~h}: 8 \mathrm{~h}$ (light/dark) photoperiod with a light intensity of $100 \mu \mathrm{mol} \mathrm{m}^{-2} \mathrm{~s}^{-1}$, after which they were subcultured every four weeks. The Gala 3 plants were rooted and transplanted as described by Zheng et al. ${ }^{39}$. Apple callus induction and culture were conducted as described by Zheng et al. ${ }^{39}$.

\section{Quantitative reverse transcription PCR (qPCR)-based analysis}

qPCR-based analysis was used to measure $M d W R K Y 11$ and $M d H M A 5$ expression in apple plants exposed to 
(a)

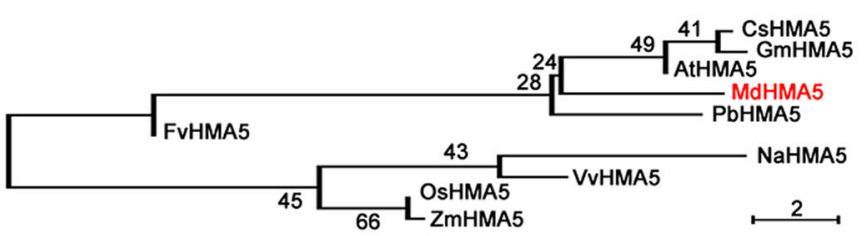

(b)

(d)

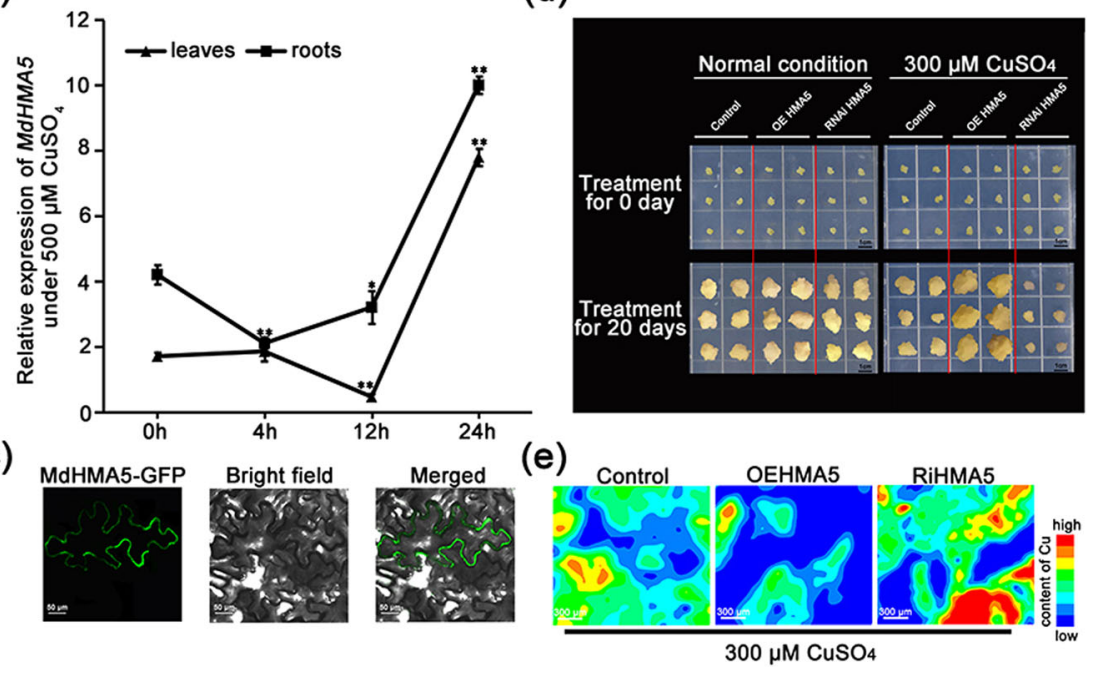

Fig. 5 Phylogenetic analysis, subcellular localization, and functional analysis of MdHMA5. a Phylogenetic tree of the HMA5 subgroup of heavy metal ATPases from select plant species. The species and the accession numbers of the amino acid sequences used in the analysis are as follows: Arabidopsis thaliana (AT1G63440), Oryza sativa (OS04G46940), Fragaria vesca (FvH4_5g02091), Vitis vinifera (XP_010651259.1), Cucumis sativus (CSPI05G05530), Zea mays (ZM2G143512), Nicotiana attenuata (XP_019241377.1), Glycine max (GM11G024400), and Pyrus x bretschneideri

(XP_018506630.1). b MdHMA5 expression in the leaves and roots under excess Cu treatment measured by qPCR and normalized according to the MdActin expression level. Hydroponic apple plants under excess Cu stress were sampled at 0, 4, 12, and 24h of treatment. The data are the means \pm SDs of triplicate experiments for each time point. The asterisks indicate the values that are significantly different from the controls (Student's $t$-test): ${ }^{*} P<0.05 ;{ }^{*} P<0.01$. c MdHMA5-GFP is localized to the plasma membrane of Nicotiana benthamiana leaf epidermal cells. 355::MdHMA5-GFP was transiently expressed in epidermal cells of $N$. benthamiana leaves and visualized by confocal microscopy $(\times 40)$. $\mathbf{d}$ Cu tolerance of transgenic

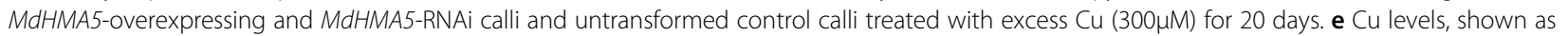
$\mu$-XRF elemental maps, in transgenic MdHMA5-overexpressing and MdHMA5-RNAi calli and control calli under excess Cu stress for 20 days

excess $\mathrm{Cu}$. Hydroponically cultivated apple plants that had 4-6 leaves and were approximately $10 \mathrm{~cm}$ tall were treated with Hoagland nutrient solution supplemented with $500 \mu \mathrm{M} \mathrm{CuSO}_{4}$. Root and leaf samples were collected at $0,1,2,4,12$, and $24 \mathrm{~h}$. Total RNA isolation, reverse transcription, and qPCR were performed as described by Zheng et al. ${ }^{39}$. MdActin was used as an internal control. MdWRKY11 and MdHMA5 expression in transgenic plants and control plants or calli was assessed similarly. All the primers used are listed in Supporting Information Table S1.

\section{Determination of MdWRKY11 subcellular localization}

The coding sequence of MdWRKY11 without the stop codon was amplified and subcloned into a pMDC83 vector to create a 35S::MdWRKY11-GFP fusion construct, which was subsequently introduced into Agrobacterium tumefaciens strain GV3101. The primers used to amplify the construct are listed in Supporting Information Table S2. The construct was infiltrated into $N$. benthamiana leaves, and GFP fluorescence in the transgenic leaves was observed by confocal microscopy $(\times 40)$ after staining with the nucleus-specific dye DAPI, as previously described by Zheng et al. ${ }^{39}$.

\section{Transcriptional activation assays in yeast}

With respect to transcriptional activation assays in yeast, the MdWRKY11 coding region without the stop codon was amplified and inserted into a pGBKT7 vector to generate a $p B D-M d W R K Y 11$ construct for MdWRKY11 expression as a fusion protein with the GAL4-binding domain (BD). The primers used are listed in Table S2. The transcriptional activation assay of MdWRKY11 was performed as described previously ${ }^{39}$. pCL-1 and pGBKT7 vectors were used as positive and negative controls, respectively. 


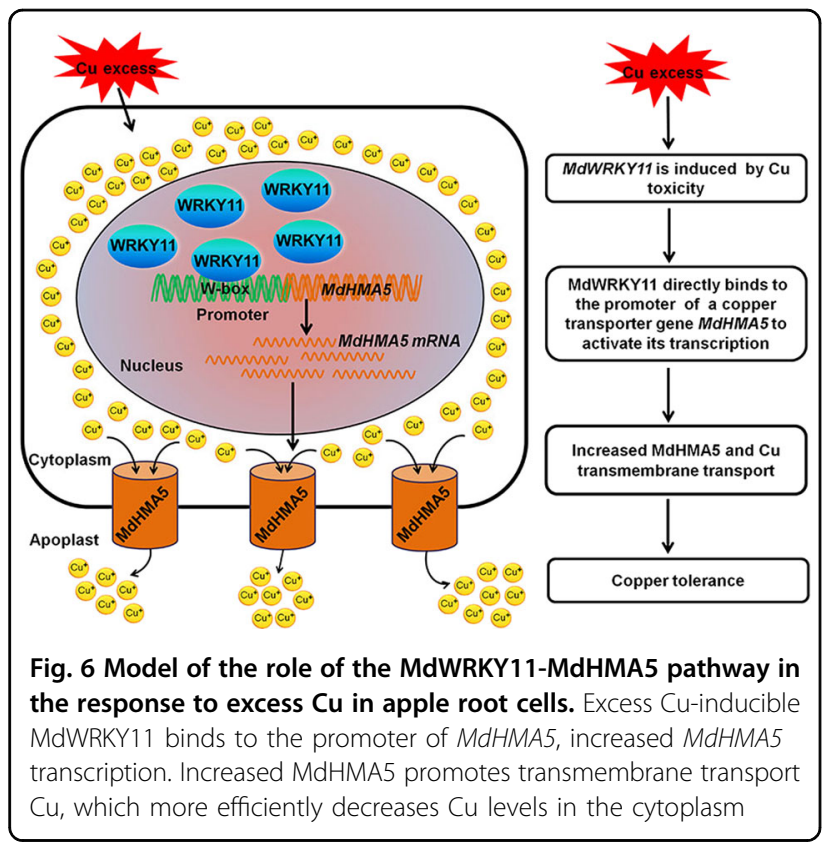

Generating transgenic Gala 3 apple plants and calli presenting altered MdWRKY11 and MdHMA5 expression

The MdWRKY11 coding region was amplified and inserted into a pBI121 vector to generate a 35S:: MdWRKY11-GUS overexpression construct. The plasmid was then introduced into Agrobacterium tumefaciens strain EHA105 for subsequent Agrobacterium-mediated transformation of Gala3 according to the method of Dai et al. $^{40}$. Transgenic plants were confirmed by PCR analysis, while the mRNA abundance of MdWRKY11 in all transgenic apple lines and control plants was determined by qPCR-based analysis. Each experiment was independently repeated three times.

To generate transgenic calli overexpressing $M d W R K Y 11$ or MdHMA5, the coding sequence of $M d W R K Y 11$ or MdHMA5 was subcloned into a pMDC83 vector to generate $35 S:: M d W R K Y 11$ and 35S::MdHMA5 plasmids, respectively. To reduce MdWRKY11 and $M d H M A 5$ expression in apple calli, the sense and antisense fragments of the two genes were inserted into a pZH01 RNA interference (RNAi) vector, yielding RNA interference constructs $p Z H 01-M d W R K Y 11-R N A i$ and pZH01-MdHMA5-RNAi, respectively. The primers used in this experiment are listed in Supporting Information Table S2. The plasmids were introduced into Agrobacterium tumefaciens strain EHA105. Apple calli were transformed using the method described by An et al. ${ }^{41}$. Transgenic calli with altered expression levels of MdWRKY11 and MdHMA5 were confirmed by PCRbased analysis, and the expression levels of $M d W R K Y 11$ or $M d H M A 5$ in all the transgenic lines and control calli were quantified via qPCR. Each experiment was independently repeated three times.

\section{Determination of the $\mathrm{Cu}$ tolerance of transgenic apple plants or calli presenting altered levels of MdWRKY11 or MdHMA5 expression}

To determine the $\mathrm{Cu}$ tolerance of transgenic apple plants, transgenic apple plants overexpressing MdWRKY11 and control plants were watered with fullstrength Hoagland nutrient solution supplemented with $500 \mu \mathrm{M} \mathrm{CuSO} \mathrm{C}_{4}$ every three days, and the $\mathrm{pH}$ of the nutrient solution was adjusted to 5.6. To apply the excess $\mathrm{Cu}$ treatment, apple calli were grown on proliferation media that consisted of $300 \mu \mathrm{M} \mathrm{CuSO}$ for 20 days. Images of the plants and calli were taken before and after treatment.

\section{Detection of $\mathrm{Cu}$ content via micro-X-ray fluorescence $(\mu-$ XRF) microspectroscopy}

The micro-X-ray fluorescence ( $\mu$-XRF) microspectroscopy experiment was performed via a 4W1B beamline system at the Beijing Synchrotron Radiation Facility (BSRF), Institute of High Energy Physics, Chinese Academy of Sciences, which runs $2.5 \mathrm{GeV}$ electrons with current from 150 to $250 \mathrm{~mA}$. The incident X-ray energy was monochromatized via a $\mathrm{W} / \mathrm{B}_{4} \mathrm{C}$ double-multilayermonochromator (DMM) at $15 \mathrm{keV}$ and was narrowed to $50 \mu \mathrm{m}$ in diameter by a polycapillary lens. After being treated with a $500 \mu \mathrm{M} \mathrm{CuSO}$ solution for $24 \mathrm{~h}$, the roots and leaves of Gala 3 plants overexpressing MdWRKY11 and those of control plants were sampled for $\mathrm{Cu}$ detection. Apple calli with altered levels of MdHMA5 expression were collected after excess $\mathrm{Cu}$ treatment on media consisting of $300 \mu \mathrm{M} \mathrm{CuSO}_{4}$ for 20 days. A cryotome was used to obtain 200-mm thick root cross-sections and calli sections, and the sections were placed on Kapton tape and freeze-dried in a vacuum freeze dryer (LGJ-10B, Beijing Four-Ring Science Instrument Factory). The sample was held on a precision motor-driven stage and scanned at 60$\mu \mathrm{m}$ intervals by two-dimensional mapping. A Si (Li) solidstate detector was used to detect XRF emission lines with a live time of $60 \mathrm{~s}$. The data were processed using the PyMCA package ${ }^{42,43}$.

\section{Heterologous MdWRKY11 expression in E. coli and preparation of polyclonal antibodies}

The coding sequence of $M d W R K Y 11$ was amplified and inserted into a pGEX-6p-1 vector. The GST-MdWRKY11 fusion protein was then expressed and purified as described by Zheng et al. ${ }^{44}$. Polyclonal anti-MdWRKY11 antibodies was prepared using the method approved by the Beijing Municipal Commission of Science and Technology 44 . 


\section{Chromatin immunoprecipitation-qPCR (ChIP-qPCR) assays}

ChIP assays involving anti-MdWRKY11 polyclonal antibodies were performed as described by Zheng et al. ${ }^{39}$. The primers for ChIP-qPCR were designed to amplify regions in the promoter sequence of MdHMA5 (Table S2). The experiment was performed in triplicate.

\section{Electrophoretic mobility shift assays (EMSAs)}

EMSAs were performed using a LightShift Chemiluminescent EMSA Kit (Thermo Scientific, Waltham, MA, USA) as described by Zheng et al. ${ }^{39}$. The $5^{\prime}$ biotin-labeled MdHMA5 promoter DNA probe containing the W-box (proMdHMA5-F-biotin + proMdHMA5-R), mutated Wbox probe (proMdHMA5 (m) -F-biotin + proMdHMA5 (m) $-\mathrm{R})$, and corresponding competitor DNA sequences (proMdHMA5-F + proMdHMA5-R) are listed in Table S2.

\section{Detection of gene expression in the transiently transformed apple calli}

To determine the effects of $M d W R K Y 11$ overexpression on MdHMA5 expression, the promoter of MdHMA5 was amplified and inserted into a pCAMBIA1301 vector to generate a proMdHMA5:: GUS construct. Apple calli were transiently cotransformed with Agrobacterium EHA105 strains carrying 35S7MdWRKY11-GFP, proMdHMA57GUS, and 35S7LUC. Apple calli cotransformed with Agrobacterium strains carrying 35S::GFP, proMdHMA5::GUS, and 35S::LUC were used as controls. The GUS and LUC activities were determined as described by Zheng et al. ${ }^{39}$. The GUS:LUC activity ratio was used as the ultimate quantification of GUS activity. Each experiment was independently repeated three times.

\section{Phylogenetic analysis}

With respect to the phylogenetic analysis of MdHMA5, previously annotated HMA5 homologs from Arabidopsis thaliana (AT1G63440), Oryza sativa (OS04G46940), Fragaria vesca (FvH4_5g02091), Vitis vinifera (XP_010651259.1), Cucumis sativus (CSPI05G05530), Zea mays (ZM2G143512), Nicotiana attenuata (XP_019241377.1), Glycine max (GM11G024400), and Pyrus x bretschneideri (XP_018506630.1) were retrieved from GenBank and aligned with MUSCLE in MEGA 7. A phylogenetic tree was then constructed using the neighbor-joining method with 1,000 bootstraps.

\footnotetext{
Acknowledgements

This research was supported by the National Key Research and Development Program of China (SQ2018YFD100303 and 2019YFD1000104), National Natural Science Fund (No. 31772279) and the Construction of Beijing science and technology innovation and service capacity in top subjects (CEFFPXM2019_014207_000032). The $\mu$-XRF beam time was granted by the 4W1B beamline of the Beijing Synchrotron Radiation Facility, Institute of High Energy Physics, Chinese Academy of Sciences. The staff members of 4W1B are acknowledged for their support in the measurements and data reduction.
}

\section{Author contributions}

J.K. planned and designed the research. K.S., X.L., Y.Z., Y.B., D.S., X.Z., L.W., H.Z., C. W., T.Y., F.Z., Z.H., Y.S., Y.G., and J.K. performed experiments, conducted the fieldwork, analyzed the data, etc. K.S. and J.K. wrote the manuscript.

\section{Conflict of interest}

The authors declare that they have no conflict of interest.

Supplementary Information accompanies this paper at (https://doi.org/ 10.1038/s41438-020-0326-0).

Received: 6 January 2020 Revised: 16 April 2020 Accepted: 20 April 2020 Published online: 01 July 2020

\section{References}

1. Li, W., Zhang, M. \& Shu, H. Distribution and fractionation of copper in soils of apple orchards. Environ. Sci. Pollut. R. 12, 168-172 (2005).

2. Liu, C. S. et al. Copper toxicity and accumulation in potted seedlings of three apple rootstock species: implications for safe fruit production on copperpolluted soils. J. Plant. Nutr. 34, 1268-1277 (2011).

3. Brun, A., Maillet, J., Hinsinger, P. \& Pépina, M. Evaluation of copper availability to plants in copper-contaminated vineyard soils. Environ. Pollut. 111, 293-302 (2001).

4. Wang, Q. Y., Liu, J. S. \& Cheng, S. Heavy metals in apple orchard soils and fruits and their health risks in Liaodong peninsula, Northeast China. Environ. Monit. Assess. 187, 4178 (2015).

5. Girotto, E. et al. Copper availability assessment of cu-contaminated vineyard soils using black oat cultivation and chemical extractants. Environ. Monit. Assess. 186, 9051-9063 (2014).

6. Adrees, M. et al. The effect of excess copper on growth and physiology of important food crops: a review. Environ. Sci. Pollut. Res. 22, 8148-8162 (2015).

7. Kan, S. H., Sun, B. Y. \& Liu, C. S. Toxic effects of long-term low-dose Copper (Cu) stress on apple trees in brown soils. J. Agro-Environ. Sci. 29, 38-42 (2010).

8. De Forest, D. K. C. \& Meyer, J. S. Critical review: toxicity of diet borne metals to aquatic organisms. Crit. Rev. Env. Sci. Tec. 45, 1176-1241 (2015).

9. Hippler, F. W. R. et al. Citrus rootstocks regulate the nutritional status and antioxidant system of trees under copper stress. Environ. Exp. Bot. 130, 42-52 (2016).

10. Leng, $X$. et al. Transporters, chaperones, and P-type ATPases controlling grapevine copper homeostasis. Funct. Integr. Genomics 15, 673-684 (2015)

11. Li, Q. et al. Excess copper effects on growth, uptake of water and nutrients, carbohydrates, and PSII photochemistry revealed by OJIP transients in Citrus seedlings. Environ. Sci. Pollut. Res. 26, 30188-30205 (2019).

12. Wang, Q. Y., Liu, J. S. \& Hu, B. Integration of copper subcellular distribution and chemical forms to understand copper toxicity in apple trees. Environ. Exp. Bot. 123, 125-131 (2016).

13. Nishizono, H., Ichikawa, H., Suzikp, S. \& Ishii, F. The role of the root cell wall in the heavy metal tolerance of Athyrium yokoscense. Plant Soil 101, 15-20 (1987).

14. Burkhead, J. L., Reynolds, K. A., Abdel-Ghany, S. E., Cohu, C. M. \& Pilon, M. Copper homeostasis. N. Phytol. 182, 799-816 (2009).

15. Kholodova, V. P., Ivanova, E. M., \& Kuznetsov, V. V. Initial steps of copper detoxification: outside and inside of the plant cell. In Detoxification of Heavy Metals, Soil Biology 30 (eds. Sherameti, I. \& Varma, A.) Ch. 8, 143-167 (SpringerVerlag Berlin Heidelberg, 2011).

16. Andres-Colas, N. et al. The Arabidopsis heavy metal P-type ATPase HMA5 interacts with metallochaperones and functions in copper detoxification of roots. Plant J. 45, 225-236 (2006).

17. Cobbett, C. S., Hussain, D. \& Haydon, M. J. Structural and functional relationships between type 1B heavy metal-transporting P-type ATPases in Arabidopsis. N. Phytol. 159, 315-321 (2003).

18. Argüello, J. M. Identification of ion-selectivity determinants in heavy-metal transport P 1B-type ATPases. J. Membr. Biol. 195, 93-108 (2003).

19. Yruela, I. Copper in plants: acquisition, transport and interactions. Funct. Plant Biol. 36, 409-430 (2009).

20. Kobayashi, Y. et al. Amino acid polymorphisms in strictly conserved domains of a P-Type ATPase HMA5 are involved in the mechanism of copper tolerance variation in Arabidopsis. Plant Physiol. 148, 969-980 (2008). 
21. Li, Y. B. et al. Two Silene vulgaris copper transporters residing in different cellular compartments confer copper hypertolerance by distinct mechanisms when expressed in Arabidopsis thaliana. N. Phytol. 215, 1102-1114 (2017).

22. Zhang, Y. Y. et al. OsATX1 interacts with heavy metal P1B-Type ATPases and affects copper transport and distribution. Plant Physiol. 178, 329-344 (2018).

23. Lange, B. et al. Copper and cobalt accumulation in plants: a critical assessment of the current state of knowledge. N. Phytol. 213, 537-551 (2017).

24. Deng, F. L., Yamaji, N., Xia, J. X. \& Ma, J. F. A member of the heavy metal P-type ATPase OsHMA5 is involved in xylem loading of copper in rice. Plant Physiol. 163, 1353-1362 (2013)

25. He, L. et al. Maize OXIDATIVE STRESS2 homologs enhance cadmium tolerance in Arabidopsis through activation of a putative SAM-dependent methyltransferase gene. Plant Physiol. 171, 1675-1685 (2016).

26. Khare, D. et al. Root avoidance of toxic metals requires the GeBP-LIKE 4 transcription factor in Arabidopsis thaliana. N. Phytol. 213, 1257-1273 (2017)

27. Lin, T. T., Yang, W. N., Lu, W., Wang, Y. \& Qi, X. T. Transcription factors PvERF15 and PVMTF-1 form a cadmium stress transcriptional pathway. Plant Physiol. 173, 1565-1573 (2017).

28. Yang, G. et al. Overexpression of ThVHAc1 and its potential upstream regulator, ThWRKY7, improved plant tolerance of cadmium stress. Sci. Rep. $\mathbf{6}$ 18752 (2016).

29. Kropat, J. et al. A regulator of nutritional copper signaling in Chlamydomonas is an SBP domain protein that recognizes the GTAC core of copper response element. Proc. Natl Acad. Sci. USA 102, 18730-18735 (2005)

30. Ruzsa, S. M. \& Scandalios, J. G. Altered Cu metabolism and differential transcription of $\mathrm{Cu} / \mathrm{ZnSod}$ genes in a Cu/ZnSOD-deficient mutant of maize: evidence for a Cu-responsive transcription factor. Biochemistry 42, 1508-1516 (2003).

31. Yamasaki, H., Hayashi, M., Fukazawa, M., Kobayashi, Y. \& Toshiharu Shikanai, T. SQUAMOSA promoter-binding protein-like 7 is a central regulator for copper homeostasis in Arabidopsis. Plant Cell 21, 347-361 (2009).

32. Ding, Z. J., Yan, J. Y., Xu, X. Y., Li, G. X. \& Zheng, S. J. WRKY46 functions as a transcriptional repressor of ALMT1, regulating aluminum-induced malate secretion in Arabidopsis. Plant J. 76, 825-835 (2013).
33. Hong, C. Y. et al. The role of ZmWRKY4 in regulating maize antioxidant defense under cadmium stress. Biochem. Bioph. Res. Commun. 482, 1504-1510 (2017).

34. Yan, J. Y. et al. A WRKY transcription factor regulates Fe translocation under Fe deficiency in Arabidopsis. Plant Physiol. 171, 2017-2027 (2016).

35. Dai, X., Wang, Y. \& Zhang, W. H. OsWRKY74, a WRKY transcription factor, modulates tolerance to phosphate starvation in rice. J. Exp. Bot. 67, 947-960 (2016).

36. Jiang, Y., Liang, G., Yang, S. \& Yu, D. Arabidopsis WRKY57 functions as a node of convergence for jasmonic acid- and auxin-mediated signaling in jasmonic acid-induced leaf senescence. Plant Cell 26, 230-245 (2014).

37. Li, W., Wang, H. \& Yu, D. Arabidopsis WRKY transcription factors WRKY12 and WRKY13 oppositely regulate flowering under short-day conditions. Mol. Plant 9, 1492-1503 (2016)

38. Iglesias, I., Echeverría, G. \& Soria, Y. Differences in fruit colour development, anthocyanin content, fruit quality and consumer acceptability of eight 'Gala' apple strains. Sci. Hortic.-Amst. 119, 32-40 (2008).

39. Zheng, X. D. et al. MdWRKY9 overexpression confers intensive dwarfing in the M26 rootstock of apple by directly inhibiting brassinosteroid synthetase MdDWF4 expression. N. Phytol. 217, 1086-1098 (2018).

40. Dai, H. et al. Development of a seedling clone with high regeneration capacity and susceptibility to Agrobacterium in apple. Sci. Hortic.-Amst. 164, 202-208 (2013).

41. An, X. H. et al. MdMYB9 and MdMYB11 are involved in the regulation of the JA-induced biosynthesis of anthocyanin and proanthocyanidin in apples. Plant Cell Physio. 56, 650-662 (2015).

42. Kim, S. A. et al. Localization of iron in Arabidopsis seed requires the vacuolar membrane transporter VIT1. Science 314, 1295-1298 (2006)

43. Solé, V. A., Papillon, E., Cotte, M., Walter, P. \& Susini, J. A multiplatform code for the analysis of energy-dispersive $X$-ray fluorescence spectra. Spectrochim. Acta B 62, 63-68 (2007).

44. Zheng, X. D. et al. Chloroplastic biosynthesis of melatonin and its involvement in protection of plants from salt stress. Sci. Rep. 7, 41236 (2017). 